\title{
A Novel Probabilistic Data Association Based MIMO Detector Using Joint Detection of Consecutive Symbol Vectors
}

\author{
Shaoshi Yang \\ School of Information and Communication Engineering \\ Beijing University of Posts and Telecommunications \\ Beijing, China \\ E-mail: banpoheitao@gmail.com
}

\begin{abstract}
A new probabilistic data association (PDA) approach is proposed for symbol detection in spatial multiplexing multiple-input multiple-output (MIMO) systems. By designing a joint detection (JD) structure for consecutive symbol vectors in the same transmit burst, more a priori information is exploited when updating the estimated posterior marginal probabilities for each symbol per iteration. Therefore the proposed PDA detector (denoted as PDA-JD detector) outperforms the conventional PDA detectors in the context of correlated input bit streams. Moreover, the conventional PDA detectors are shown to be a special case of the PDA-JD detector. Simulations and analyses are given to demonstrate the effectiveness of the new method.
\end{abstract}

Index Terms-Gaussian approximation, MIMO detection, maximum likelihood (ML) detection, probabilistic data association (PDA)

\section{INTRODUCTION}

$\mathrm{T}$ he probabilistic data association (PDA) algorithm is a reduced complexity approximation to the a posteriori probability (APP) detector, and has attracted great interests for suboptimal detection of CDMA [1] and spatial multiplexing multiple-input multiple-output (MIMO) systems [2]-[4].

It is pointed out in [5] that if the information bits represented by constellation symbols are uncoded, then decisions on the symbols can be made by any feasible MIMO detector on a vector-by-vector basis. However, if the MIMO detector is confronted by symbols that are correlated through some form of channel code, which is usually a more practical case, it is suboptimal for the signal detector and channel decoder to operate separately and only on individual symbol vectors. To optimally detect the symbols, the detector should make decisions jointly on all the symbol vectors using knowledge of the correlations across symbol vectors introduced by the channel code, and the channel code should decode using likelihood information on all the symbol vectors obtained from the signal detector. However, this is computationally prohibitive.

Therefore, the suboptimal solutions are often applied, including the powerful iterative receiver that combines MIMO detector and a soft-input soft-output channel decoder to accomplish joint detection and decoding (see Fig. 1 [6]), or more simplified scheme where MIMO detector and channel

\author{
Tiejun Lv \\ School of Information and Communication Engineering \\ Beijing University of Posts and Telecommunications \\ Beijing, China \\ E-mail: lvtiejun@gmail.com
}

decoder are combined in a straightforward sequential style without feedback (see Fig. 2). For research of detector, it is general to focus on parts inside the dashed frames.

Since its output is probabilistic information, PDA algorithm is appropriate to play the role of "soft MIMO detector" in both the iterative and straightforward sequential receivers.

Despite relatively favorable tradeoff between performance and computational complexity, the conventional PDA detectors suffer intrinsic error compared with ML detector, rather than it has previously been claimed that PDA can avoid local minima [1]. The key feature of the conventional PDA algorithms in [1]-[4] is a repeated conversion of a multimodal Gaussian mixture probability structure, the true probability function for the noise and interference, to a single Gaussian with matched mean and covariance. Consequently, an implicit gap exists between the "Gaussian approximation" and the Gaussian mixture especially when the number of transmit antennas is small [7], with the result that the conventional PDA detectors are not generally near optimal. This is demonstrated by the simulation results for PDA under different modulation schemes in [1][2] and a theoretical proof in [8]. In addition PDA algorithm proceeds in iterative manner, thus some potential measures may be utilized to further enhance the performance of conventional PDA detectors under certain circumstances.

In this paper, we propose a new PDA based MIMO detector that achieves joint detection (JD) of consecutive symbol vectors in the same transmit burst, instead of the conventional strategy detecting the symbol vector in a burst one by one. The proposed PDA-JD detector exploits statistical correlations between consecutive symbol vectors, and leads to superior performance to conventional PDA MIMO detectors in the context of correlated input data symbols. In addition, the complexity of PDA-JD detector is shown to be polynomial as similar to that of conventional PDA algorithms. The improved quality of soft reliability information provided for the concatenated channel decoder by PDA-JD detector can not only help reduce the complexity of both the iterative and the straightforward sequential receivers, but also abate error propagation in the latter scheme.

This research has been supported by Program for New Century Excellent Talents in University (NCET) under Grant NCET-06-0091. 


\section{BASIC SYSTEM MODEL}

Consider a spatial multiplexing MIMO system with $N_{T}$ transmit antennas and $N_{R} \geq N_{T}$ receive antennas (extending the proposed algorithm for $N_{R}<N_{T}$ is a straightforward matter of adopting the non-decorrelated system model in [7] to the current problem). The received baseband signal at each instant of time is given by

$$
\mathbf{y}=\mathbf{H x}+\mathbf{n},
$$

where $\mathbf{H}$ is the $N_{R} \times N_{T}$ channel matrix, $\mathbf{x}$ is the length $N_{T}$ vector of transmit symbols taken from a modulation constellation $A=\left\{a_{1}, a_{2}, \cdots a_{M}\right\}$ with cardinality $M$, and $\mathbf{n}$ is a length $N_{R}$ zero mean complex circular symmetric Gaussian noise vector with covariance matrix $\sigma^{2} \mathbf{I}$, where $\mathbf{I}$ is an $N_{R} \times N_{R}$ identity matrix.

At the receiver, when the PDA-based algorithms are considered, the task of symbol detection is to estimate the posterior marginal symbol probabilities $\tilde{P}\left(x_{j}=a_{m} \mid \mathbf{y}\right)=\tilde{P}_{m}\left(x_{j} \mid \mathbf{y}\right)$ for $j=1,2, \cdots, N_{T}, m=1,2, \cdots, M$ without making an exhaustive search in the space of all possible symbol combinations.

Based on (1), the decorrelated system model for PDA detectors is formulated as

$$
\tilde{\mathbf{y}}=\mathbf{x}+\tilde{\mathbf{n}}=x_{j} \mathbf{e}_{j}+\sum_{k \neq j} x_{k} \mathbf{e}_{k}+\tilde{\mathbf{n}} \triangleq x_{j} \mathbf{e}_{j}+\tilde{\mathbf{N}}_{j},
$$

where $\tilde{\mathbf{y}}=\left(\mathbf{H}^{H} \mathbf{H}\right)^{-1} \mathbf{H}^{H} \mathbf{y}, \tilde{\mathbf{n}}$ is a colored Gaussian noise with zero mean and covariance $\sigma^{2}\left(\mathbf{H}^{H} \mathbf{H}\right)^{-1}, \mathbf{e}_{k}$ is a column vector with 1 in the $k$ th position and 0 elsewhere, and $\tilde{\mathbf{N}}_{j}$ is the interference and noise term.

\section{PDA-JD MIMO DETECTOR}

\section{A. On JD of Consecutive Symbol Vectors}

Note that there are generally hundreds of symbol vectors in a single transmission burst of MIMO systems [10]. These symbol vectors are all received in a time interval as a block. Suppose the number of symbol vectors in a burst is denoted as $L$, then corresponding to each transmitted symbol vector, the received signals are $\mathbf{y}^{(1)}=\mathbf{H}^{(1)} \mathbf{x}^{(1)}+\mathbf{n}^{(1)}, \mathbf{y}^{(2)}=\mathbf{H}^{(2)} \mathbf{x}^{(2)}+\mathbf{n}^{(2)}, \cdots$, $\mathbf{y}^{(l)}=\mathbf{H}^{(l)} \mathbf{x}^{(l)}+\mathbf{n}^{(l)} \quad, \quad l=1,2, \cdots, L \quad, \quad$ respectively. Conventionally the received symbol vectors are detected on an isolated vector-by-vector basis even in coded system. However, while soft information and correlations across symbol vectors introduced by some form of channel code are taken into consideration, to detect $\mathbf{x}^{(1)}, \mathbf{x}^{(2)}, \cdots$ optimally, the detector should compute the likelihood of each symbol given all the received symbol vectors $\mathbf{y}^{(1)}, \mathbf{y}^{(2)}, \cdots$. Although this is computationally prohibitive, it is still valuable to make a tradeoff between the enhanced performance and complexity from the integral system point of view.

Our idea is to jointly detect an appropriate number of consecutive symbol vectors. To our best knowledge, such a detection strategy in MIMO systems has not been reported before.

We assume that the number of symbol vectors detected simultaneously is denoted as $d$, which satisfies $d \in\{1,2, \cdots, L\} \cap N d=L$, with $N$ being a positive integer. As a result, the system model (1) is redesigned as

$$
\mathbf{r}=\mathbf{G s}+\mathbf{u}
$$

where $\mathbf{r}=\left[\left(\mathbf{y}^{(1)}\right)^{T}, \cdots,\left(\mathbf{y}^{(d)}\right)^{T}\right]^{T}, \mathbf{s}=\left[\left(\mathbf{x}^{(1)}\right)^{T}, \cdots,\left(\mathbf{x}^{(d)}\right)^{T}\right]^{T}$,

$\mathbf{u}=\left[\left(\mathbf{n}^{(1)}\right)^{T}, \cdots,\left(\mathbf{n}^{(d)}\right)^{T}\right]^{T}, \mathbf{G}=\left(\begin{array}{ccc}\mathbf{H}^{(1)} & & \mathbf{0} \\ & \ddots & \\ \mathbf{0} & & \mathbf{H}^{(d)}\end{array}\right)$, and $\mathbf{u}$ is

still a zero mean complex circular symmetric Gaussian noise vector with covariance matrix

$\operatorname{Cov}(\mathbf{u})=\mathrm{E}\left(\mathbf{u u}^{H}\right)=\left(\begin{array}{ccc}\sigma_{1}^{2} \mathbf{I} & & \mathbf{0} \\ & \ddots & \\ \mathbf{0} & & \sigma_{d}^{2} \mathbf{I}\end{array}\right)$, in which $\sigma_{d^{\prime}}^{2}$ is the power of the white noise $\mathbf{n}^{\left(d^{\prime}\right)}$ corresponding to the $d^{\prime}$ th symbol vector, $d^{\prime}=1,2, \cdots, d$.

Similarly to (2), (3) can be reformulated as the decorrelated model

$$
\tilde{\mathbf{r}}=\mathbf{s}+\tilde{\mathbf{u}}=s_{j^{\prime}} \mathbf{e}_{j^{\prime}}+\sum_{k^{\prime} \neq j^{\prime}} s_{k^{\prime}} \mathbf{e}_{k^{\prime}}+\tilde{\mathbf{u}}=s_{j^{\prime}} \mathbf{e}_{j^{\prime}}+\tilde{\mathbf{U}}_{j^{\prime}},
$$

where

$\tilde{\mathbf{r}}=\left(\mathbf{G}^{H} \mathbf{G}\right)^{-1} \mathbf{G}^{H} \mathbf{r}$

$=\left(\left(\left(\mathbf{H}^{(1) H} \mathbf{H}^{(1)}\right)^{-1} \mathbf{H}^{(1) H} \mathbf{y}^{(1)}\right)^{T}, \cdots,\left(\left(\mathbf{H}^{(d) H} \mathbf{H}^{(d)}\right)^{-1} \mathbf{H}^{(d) H} \mathbf{y}^{(d)}\right)^{T}\right)^{T}$

, $\tilde{\mathbf{u}}$ is Gaussian noise with zero mean and covariance

$$
\begin{aligned}
& \operatorname{Cov}(\tilde{\mathbf{u}})=\left(\mathbf{G}^{H} \mathbf{G}\right)^{-1} \mathbf{G}^{H}\left(\begin{array}{ccc}
\sigma_{1}^{2} \mathbf{I} & & \mathbf{0} \\
& \ddots & \\
\mathbf{0} & & \sigma_{d}^{2} \mathbf{I}
\end{array}\right) \mathbf{G}\left(\mathbf{G}^{H} \mathbf{G}\right)^{-1} \\
& =\left(\begin{array}{ccc}
\sigma_{1}^{2}\left(\mathbf{H}^{(1) H} \mathbf{H}^{(1)}\right)^{-1} & & \mathbf{0} \\
& \ddots & \\
\mathbf{0} & & \sigma_{d}^{2}\left(\mathbf{H}^{(d) H} \mathbf{H}^{(d)}\right)^{-1}
\end{array}\right),
\end{aligned}
$$

$j^{\prime}, k^{\prime}=1,2, \cdots, d \times N_{T}$. Since the elements of $\mathbf{H}^{\left(d^{\prime}\right)}$ are i.i.d. complex Gaussian, the rank of $\mathbf{H}^{\left(d^{\prime}\right)}$ is almost always $N_{T}$, i.e. $\mathbf{H}^{\left(d^{\prime}\right)}$ has full column rank. So the Hermitian matrix $\mathbf{H}^{\left(d^{\prime}\right) H} \mathbf{H}^{\left(d^{\prime}\right)}$ is almost definitely invertible.

Notice that the above derivation does not make the assumption that $\mathbf{H}$ is quasi-static, which means the derivation makes sense for the fast variant channel as well where $\mathbf{H}$ changes randomly from one symbol vector to another. When quasi-static channel is taken into account, we have $\mathbf{H}^{(1)}=\mathbf{H}^{(2)}=\cdots=\mathbf{H}^{(d)}=\mathbf{H}$. Then $\tilde{\mathbf{r}}$ and $\operatorname{Cov}(\tilde{\mathbf{u}})$ are rewritten respectively as

$$
\left(\left(\left(\mathbf{H}^{H} \mathbf{H}\right)^{-1} \mathbf{H}^{H} \mathbf{y}^{(1)}\right)^{T}, \cdots,\left(\left(\mathbf{H}^{H} \mathbf{H}\right)^{-1} \mathbf{H}^{H} \mathbf{y}^{(d)}\right)^{T}\right)^{T}
$$


and $\left(\begin{array}{ccc}\sigma_{1}^{2}\left(\mathbf{H}^{H} \mathbf{H}\right)^{-1} & & \mathbf{0} \\ & \ddots & \\ \mathbf{0} & & \sigma_{d}^{2}\left(\mathbf{H}^{H} \mathbf{H}\right)^{-1}\end{array}\right)$. Furthermore, if the

Gaussian noise $\mathbf{n}^{\left(d^{\prime}\right)}$ is considered i.i.d. between different $d^{\prime}$, $\operatorname{Cov}(\tilde{\mathbf{u}})$ can be simplified as

$$
\left(\begin{array}{ccc}
\sigma^{2}\left(\mathbf{H}^{H} \mathbf{H}\right)^{-1} & & \mathbf{0} \\
& \ddots & \\
\mathbf{0} & & \sigma^{2}\left(\mathbf{H}^{H} \mathbf{H}\right)^{-1}
\end{array}\right) .
$$

From (4) we can clearly see that when any symbol $s_{j^{\prime}}$ is detected, the dimension of the corresponding interference is $d \times N_{T}-1$ instead of $N_{T}-1$ in the conventional PDA detectors. And the conventional PDA detectors are a special case of the PDA-JD algorithm when $d=1$.

\section{B. PDA Algorithm Derivation Based on JD}

For any element $s_{j^{\prime}}$, we associate a probability vector $\tilde{\mathbf{P}}\left(j^{\prime}\right)$ whose $m$ th element $\tilde{P}_{m}\left(s_{j^{\prime}} \mid \mathbf{r}\right)$, is the current estimate of the posterior probability that $s_{j^{\prime}}=a_{m}, m=1,2, \cdots, M$. Following the PDA principle ("Gaussian approximation") in [4], the pseudo noise $\tilde{\mathbf{U}}_{j^{\prime}}$ is approximated by a complex Gaussian distribution with matched mean $\sum_{k^{\prime} \neq j^{\prime}} \tilde{s}_{k^{\prime}} \mathbf{e}_{k^{\prime}}$, covariance matrix $\mathbf{\Omega}_{j^{\prime}} \triangleq \sum_{k^{\prime} \neq j^{\prime}} \omega_{k^{\prime}} \mathbf{e}_{k^{\prime}} \mathbf{e}_{k^{\prime}}^{T}+\operatorname{Cov}(\tilde{\mathbf{u}})$ and pseudo-covariance matrix $\boldsymbol{\Xi}_{j^{\prime}} \triangleq \sum_{k^{\prime} \neq j^{\prime}} \xi_{k^{\prime}} \mathbf{e}_{k^{\prime}} \mathbf{e}_{k^{\prime}}^{T}$ where

$$
\begin{gathered}
\tilde{s}_{k^{\prime}}=\sum_{m=1}^{M} a_{m} \tilde{P}_{m}\left(s_{k^{\prime}} \mid \mathbf{r}\right) \\
\omega_{k^{\prime}}=\sum_{m=1}^{M}\left(a_{m}-\tilde{s}_{k^{\prime}}\right)\left(a_{m}-\tilde{s}_{k^{\prime}}\right)^{*} \tilde{P}_{m}\left(s_{k^{\prime}} \mid \mathbf{r}\right) \\
\xi_{k^{\prime}}=\sum_{m=1}^{M}\left(a_{m}-\tilde{s}_{k^{\prime}}\right)\left(a_{m}-\tilde{s}_{k^{\prime}}\right)^{T} \tilde{P}_{m}\left(s_{k^{\prime}} \mid \mathbf{r}\right) .
\end{gathered}
$$

For more information about pseudo-covariance, see [9].

Here $\tilde{P}_{m}\left(s_{k^{\prime}} \mid \mathbf{r}\right)$ is initialized as a uniform distribution and will be replaced with an updated value where the pseudo-covariance typically does not vanish.

$$
\begin{aligned}
& \text { Let } \mathbf{w}=\tilde{\mathbf{r}}-s_{j^{\prime}} \mathbf{e}_{j^{\prime}}-\sum_{k^{\prime} \neq j^{\prime}} \tilde{s}_{k^{\prime}} \mathbf{e}_{k^{\prime}} \text { and } \\
& \qquad \begin{aligned}
\phi_{m}\left(s_{j^{\prime}}\right) \triangleq \exp \left(-\left(\begin{array}{cc}
\mathfrak{R}(\mathbf{w}) \\
\mathfrak{I}(\mathbf{w})
\end{array}\right)^{T} \boldsymbol{\Lambda}_{j^{\prime}}\left(\begin{array}{c}
\mathfrak{R}(\mathbf{w}) \\
\mathfrak{I}(\mathbf{w})
\end{array}\right)\right), \\
\boldsymbol{\Lambda}_{j^{\prime}} \triangleq\left(\begin{array}{cc}
\mathfrak{R}\left(\boldsymbol{\Omega}_{j^{\prime}}+\boldsymbol{\Xi}_{j^{\prime}}\right) & -\mathfrak{I}\left(\boldsymbol{\Omega}_{j^{\prime}}-\boldsymbol{\Xi}_{j^{\prime}}\right) \\
\mathfrak{I}\left(\mathbf{\Omega}_{j^{\prime}}+\boldsymbol{\Xi}_{j^{\prime}}\right) & \mathfrak{R}\left(\mathbf{\Omega}_{j^{\prime}}-\boldsymbol{\Xi}_{j^{\prime}}\right)
\end{array}\right) \\
=\left(\left(\begin{array}{ll}
\mathfrak{R}(\operatorname{Cov}(\tilde{\mathbf{u}})) & -\mathfrak{I}(\operatorname{Cov}(\tilde{\mathbf{u}})) \\
\mathfrak{I}(\operatorname{Cov}(\tilde{\mathbf{u}})) & \mathfrak{R}(\operatorname{Cov}(\tilde{\mathbf{u}}))
\end{array}\right)+\mathbf{Q}\right)^{-1},
\end{aligned}
\end{aligned}
$$

where $\mathfrak{R}(\bullet)$ and $\mathfrak{I}(\bullet)$ are the real and imaginary part of a complex variable respectively, and $\begin{aligned} \mathbf{Q}= & \sum_{k^{\prime} \neq j^{\prime}}\left(\omega_{k^{\prime}}+\mathfrak{R}\left(\xi_{k^{\prime}}\right)\right) \mathbf{e}_{k^{\prime}} \mathbf{e}_{k^{\prime}}^{T}+\sum_{k^{\prime} \neq j^{\prime}}\left(\omega_{k^{\prime}}-\mathfrak{R}\left(\xi_{k^{\prime}}\right)\right) \mathbf{e}_{k^{\prime}+d \times N_{T}} \mathbf{e}_{k^{\prime}+d \times N_{T}}^{T} \\ & +\sum_{k^{\prime} \neq j^{\prime}} \mathfrak{I}\left(\xi_{k^{\prime}}\right) \mathbf{e}_{k^{\prime}} \mathbf{e}_{k^{\prime}+d \times N_{T}}^{T}+\sum_{k^{\prime} \neq j^{\prime}} \mathfrak{I}\left(\xi_{k^{\prime}}\right) \mathbf{e}_{k^{\prime}+d \times N_{T}} \mathbf{e}_{k^{\prime}}^{T}\end{aligned}$.

Since it is assumed that the transmitted symbols have equal $a$ priori probabilities, the estimated posterior marginal symbol probability is given as

$$
\tilde{P}_{m}\left(s_{j^{\prime}} \mid \tilde{\mathbf{r}}\right)=\frac{\tilde{p}_{m}\left(\tilde{\mathbf{r}} \mid s_{j^{\prime}}\right) P\left(s_{j^{\prime}}=a_{m}\right)}{\sum_{m=1}^{M} \tilde{p}_{m}\left(\tilde{\mathbf{r}} \mid s_{j^{\prime}}\right) P\left(s_{j^{\prime}}=a_{m}\right)} \approx \frac{\phi_{m}\left(s_{j^{\prime}}\right)}{\sum_{m=1}^{M} \phi_{m}\left(s_{j^{\prime}}\right)} .
$$

To sum up, the algorithm proceeds as follows.

1) Determine the optimal detection sequence according to the ordering criterion proposed for the decision feedback detector in [11], and denote the sequence as $\left\{n_{j^{\prime}}\right\}_{j^{\prime}=1}^{d \times N_{T}}$.

2) Initialization: compute $\tilde{\mathbf{r}}$, set $\tilde{P}_{m}\left(s_{j^{\prime}} \mid \mathbf{r}\right)$ as a uniform distribution for $\forall j^{\prime}=1,2, \cdots, d \times N_{T}, \forall m=1,2, \cdots, M$, i.e. $\tilde{P}_{m}\left(s_{j^{\prime}} \mid \mathbf{r}\right)=1 / M$; set the iteration counter $z=1$.

3) Initialize $j^{\prime}=1$.

4) Based on the current values of $\left\{\tilde{\mathbf{P}}\left(n_{k^{\prime}}\right)\right\}_{n_{k^{\prime}} \neq n_{j^{\prime}}}$, compute $\tilde{P}_{m}\left(s_{n_{f^{\prime}}} \mid \mathbf{r}\right)$ via $(5) \sim(10)$, and set the results equal to the corresponding elements of $\tilde{\mathbf{P}}\left(n_{j^{\prime}}\right)$.

5) If $j^{\prime}<d \times N_{T}$, let $j^{\prime}=j^{\prime}+1$ and go to step 4). Otherwise, go to step 6).

6) If $\forall j^{\prime}, \tilde{\mathbf{P}}\left(j^{\prime}\right)$ has converged, or the iteration counter has come to a certain given number, go to step 7). Otherwise, let $z=z+1$ and return to step 3).

7) For $j^{\prime}=1,2, \cdots, d \times N_{T}$, make a decision $\hat{s}_{j^{\prime}}$ for $s_{j^{\prime}}$ via

$\hat{s}_{j^{\prime}}=a_{l}, l=\arg \max _{m^{\prime}=1,2, \cdots, M}\left\{\tilde{P}_{m^{\prime}}\left(s_{j^{\prime}} \mid \mathbf{r}\right)\right\}$.

Then $\hat{\mathbf{s}}=\left\{\hat{s}_{j^{\prime}} \mid j^{\prime}=1,2, \cdots, d \times N_{T}\right\}$ is obtained.

8) Finally, we have

$\mathbf{x}^{\left(d^{\prime}\right)}=\left\{\hat{s}_{j^{\prime}} \mid j^{\prime}=\left(d^{\prime}-1\right) \times N_{T}+1,\left(d^{\prime}-1\right) \times N_{T}+2, \cdots, d^{\prime} \times N_{T}\right\}$, $d^{\prime}=1,2, \cdots, d$.

From the above algorithm procedure, we can get the perspective that the essence of the PDA-JD detector is it exploits more a priori information when estimating the posterior marginal probabilities of symbols being detected. One needs to compute at least $d \times N_{T} \times(M-1)$ probabilities in each iteration. For the sake of clarity, they are depicted in Table I.

Because we are interested in the fundamental property of the PDA-JD detector, the refinements such as complexity reduction techniques proposed in [1], and conversion of the complex-valued signal model into an equivalent real-valued signal model for QAM constellation in [2], are not incorporated into the above basic algorithm procedure, though they both make sense in this PDA-JD detector. 
TABLE I

PROBABILITIES COMPUTED IN ONE ITERATION

\begin{tabular}{c|cccccc}
\hline & 1 & 2 & $\cdots$ & $m$ & $\cdots$ & $M$ \\
\hline$\tilde{\mathbf{P}}(1)$ & $\tilde{P}_{1}\left(s_{1} \mid \mathbf{r}\right)$ & $\tilde{P}_{2}\left(s_{1} \mid \mathbf{r}\right)$ & $\cdots$ & $\tilde{P}_{m}\left(s_{1} \mid \mathbf{r}\right)$ & $\cdots$ & $\tilde{P}_{M}\left(s_{1} \mid \mathbf{r}\right)$ \\
$\tilde{\mathbf{P}}(2)$ & $\tilde{P}_{1}\left(s_{2} \mid \mathbf{r}\right)$ & $\tilde{P}_{2}\left(s_{2} \mid \mathbf{r}\right)$ & $\cdots$ & $\tilde{P}_{m}\left(s_{2} \mid \mathbf{r}\right)$ & $\cdots$ & $\tilde{P}_{M}\left(s_{2} \mid \mathbf{r}\right)$ \\
$\vdots$ & $\vdots$ & $\vdots$ & $\cdots$ & $\vdots$ & $\cdots$ & $\vdots$ \\
$\tilde{\mathbf{P}}\left(j^{\prime}\right)$ & $\tilde{P}_{1}\left(s_{j^{\prime}} \mid \mathbf{r}\right)$ & $\tilde{P}_{2}\left(s_{j^{\prime}} \mid \mathbf{r}\right)$ & $\cdots$ & $\tilde{P}_{m}\left(s_{j^{\prime}} \mid \mathbf{r}\right)$ & $\cdots$ & $\tilde{P}_{M}\left(s_{j^{\prime}} \mid \mathbf{r}\right)$ \\
$\vdots$ & $\vdots$ & $\vdots$ & $\cdots$ & $\vdots$ & $\cdots$ & $\vdots$ \\
$\tilde{\mathbf{P}}\left(d \times N_{T}\right)$ & $\tilde{P}_{1}\left(s_{d \times N_{T}} \mid \mathbf{r}\right)$ & $\tilde{P}_{2}\left(s_{d \times N_{T}} \mid \mathbf{r}\right)$ & $\cdots$ & $\tilde{P}_{m}\left(s_{d \times N_{T}} \mid \mathbf{r}\right)$ & $\cdots$ & $\tilde{P}_{M}\left(s_{d \times N_{T}} \mid \mathbf{r}\right)$ \\
& & & & & & \\
\hline
\end{tabular}

\section{Complexity Analysis}

In the aspect of complexity, the key step of PDA-based algorithms is the continual calculations and updates of $\boldsymbol{\Lambda}_{j^{\prime}}$, i.e. the inverse of the "complete-covariance matrix".

Taking QAM modulation as an example, the complexity of the proposed PDA-JD detector using the matrix speed-up tactic (the Sherman-Morrison-Woodbury formula) of [1] is approximately $O\left(\left(2 d N_{T}\right)^{3} / d\right)=O\left(8 d^{2} N_{T}{ }^{3}\right)$ real operations for updating the inverse of the "complete-covariance matrix" per symbol vector per iteration. This is actually the worst case complexity, since the size of the problem may be lowered by the use of successive cancellation [1].

By comparison, the corresponding complexity is approximately $O\left(N_{T}^{3}\right)$ and $O\left(\left(2 N_{T}\right)^{3}\right)=O\left(8 N_{T}{ }^{3}\right)$ [2][4] real operations when BPSK and QAM modulation is used respectively. In [4] the author merely gives a rough result $O\left(N_{T}{ }^{3}\right)$, which is not accurate enough for the complexity comparison here.

It is clear that $N_{T}$ plays a dominant role in the complexity of all PDA based MIMO detectors, especially for the system with large $N_{T}$. Note that the error performance of the PDA-JD detector is determined by $d \times N_{T}$, and the proper $d$ is determined by the constraint length of specific channel code scheme. Therefore the PDA-JD detector enjoys almost the same order polynomial complexity of $N_{T}{ }^{3}$ as the conventional PDA detectors do, when $d$ is properly determined to be an affordable constant coefficient.

\section{SimULATION RESULTS AND DISCUSSIONS}

\section{A. Simulation Results}

In this section, we demonstrate the power of the PDA-JD detector using computer simulations for a V-BLAST system which utilizes $N_{T}=N_{R}=2$ antennas and QPSK modulation scheme. The entries of the MIMO channel are chosen as i.i.d., zero mean, standard complex normal random variables, which remain constant for the duration of the burst with length $L=64$ but change randomly from one burst to another. A rate $1 / 2$ convolutional code with polynomials $(177,133)$ is applied to introduce correlation across symbols. In order to demonstrate the error probability improvement brought by PDA-JD detector,

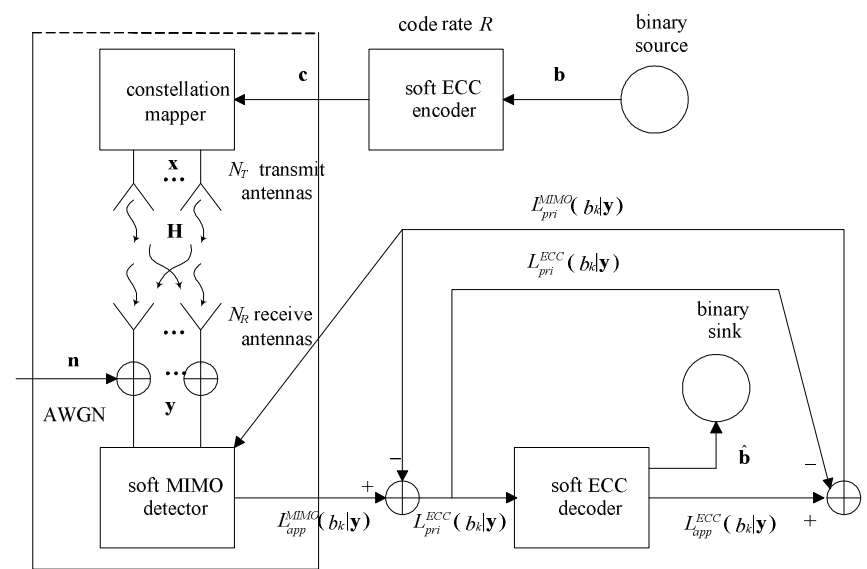

Fig. 1. Transmitter, MIMO channel, and receiver with iterative detection and decoding

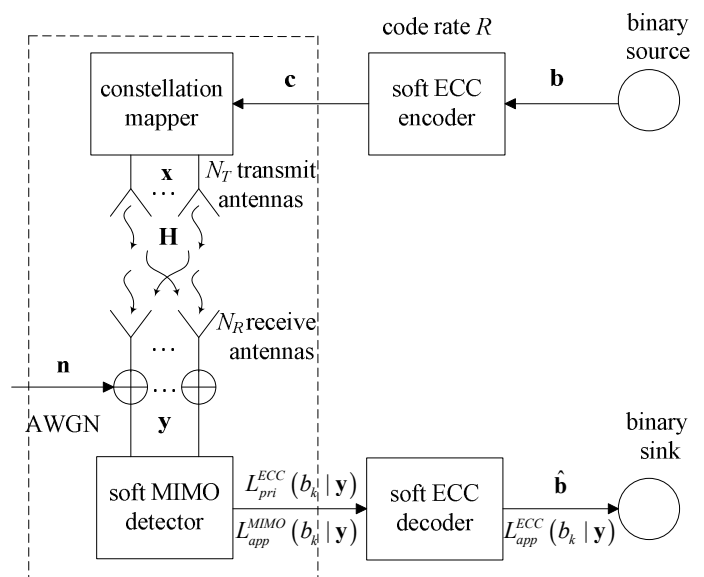

Fig. 2. Transmitter, MIMO channel, and receiver with straightforward concatenated detection and decoding

which is far less obvious when entangled with channel decoder, we focus on parts of the dashed frames in Fig. 1 and Fig. 2 and evaluate the bit error performance at the output of PDA-JD detector directly. This is a generally accepted manner in research of MIMO detectors.

The performances of the PDA-JD detector, the conventional PDA detector proposed in [4] and the ML detector are compared in Fig. 3. Both the PDA detectors do not use the optimal detection sequence and refinements we have mentioned in the above section. This is done in order to provide the same comparison conditions as in [4]. It is easy to find the advantage of the PDA-JD detector over the conventional PDA algorithm. When $d=8$ and $d=2$, the BER performance of the proposed PDA-JD detector is nearly $2 \mathrm{~dB}$ and more than $1 \mathrm{~dB}$ better than that of the conventional PDA detector respectively at $\mathrm{BER}=10^{-3}$. Unfortunately, it can observed that unlike the reported performance of the PDA in BPSK systems, in QPSK, the ML detector is still considerably better than the PDA based detectors.

Fig. 4 shows the complexity of the PDA-JD and the conventional PDA detectors (the special case of $d=1$ in the PDA-JD detector). It is evident that in both detectors $N_{T}$ imposes dominant influence on the complexity. This observation is consistent with the theoretical result 
$O\left(8 d^{2} N_{T}{ }^{3}\right)$. and $O\left(8 N_{T}{ }^{3}\right)$. Taking the constraint length 7 of $(177,133)$ into account, the more appropriate value of $d$ is 2 , which gives more favorable tradeoff between the BER performance and the computational complexity than other values of $d$ under the specific MIMO system parameters here.

\section{B. Some Discussions}

The improved error probability provided by PDA-JD detector is salient to success of both the receivers shown in Fig. 1 and Fig. 2. The iterative joint detection and decoding receiver in Fig. 1 goes in outer iteration plus inner iteration manner, where the inner iteration dwells in channel decoder itself and the outer iteration accomplishes exchanges of extrinsic information between the soft MIMO detector and the channel decoder. High-quality probabilistic data offered by soft MIMO detector like PDA-JD detector is capable of giving more reliable a priori information to its following channel decoder in both receivers in Fig. 1 and Fig. 2, and thus helps reduce the total complexity of the whole receiver through round by round iterations.

Additionally, for the straightforward concatenated receiver, the soft error decisions made by the soft MIMO detector flow into the after channel decoder and induce serious error propagation. However, by using the PDA-JD detector, this problem can be mitigated.

In fact, PDA based algorithms fall into the category of "belief-propagation" based iterative methods and most of the algorithms in this category have not yet been well understood. The performance analysis and convergence property of PDA based algorithms are still open problems and will be addressed in our future research.

\section{CONCLUSION}

We proposed a novel PDA detector using joint detection of consecutive symbol vectors in the same transmit burst for spatial multiplexing MIMO systems. Our analyses and computer simulations show that the PDA-JD detector significantly outperforms the conventional PDA detector especially in high SNR regimes in the context of correlated input bit streams, and its worst-case complexity is almost the same order polynomial of $N_{T}{ }^{3}$ as that of the conventional PDA detectors do. In addition the conventional PDA detectors are in fact a special case of the PDA-JD detector.

\section{REFERENCES}

[1] J. Luo, K. R. Pattipati, P. K. Willett, and F. Hasegawa, "Near optimal multiuser detection in synchronous CDMA using probabilistic data association," IEEE Commun. Lett., Vol. 5, No. 9, pp. 361-363, 2001.

[2] D. Pham, K. R. Pattipati, P. K. Willet, and J. Luo, "A generalized probabilistic data association detector for multiple antenna systems," IEEE Commun. Lett., Vol. 8, No. 4, pp. 205-207, 2004.

[3] S. Liu and Z. Tian, "Near-optimum soft decision equalization for frequency selective MIMO channels," IEEE Trans. On Signal Process., Vol. 52, No. 3, pp. 721-733, 2004.

[4] Y. Jia, C.M. Vithanage, C. Andrieu and R.J. Piechocki, "Probabilistic data association for symbol detection in MIMO systems," Electron. Lett. Vol. 42, No. 1, pp. 38-40, 5th Jan. 2006.

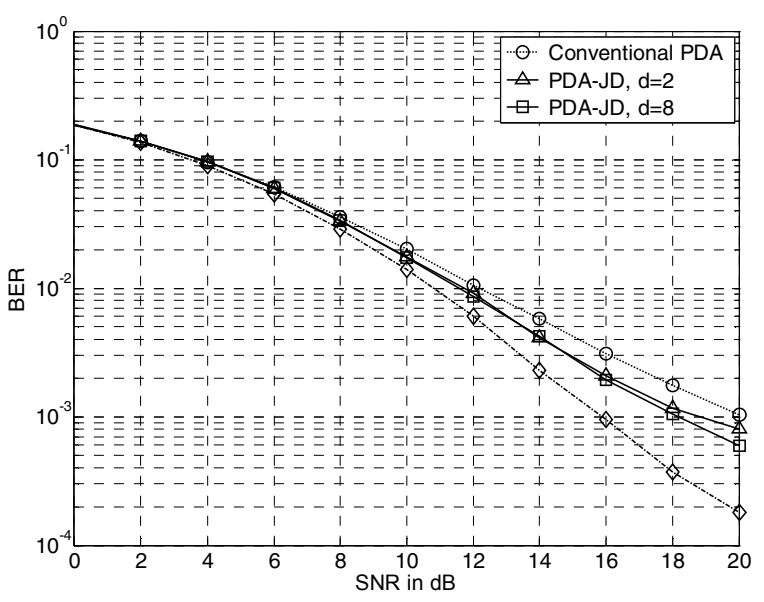

Fig. 3. Comparison of bit error rates of the PDA-JD, the conventional PDA, and the ML detectors for V-BLAST with QPSK and $N_{T}=N_{R}=2$

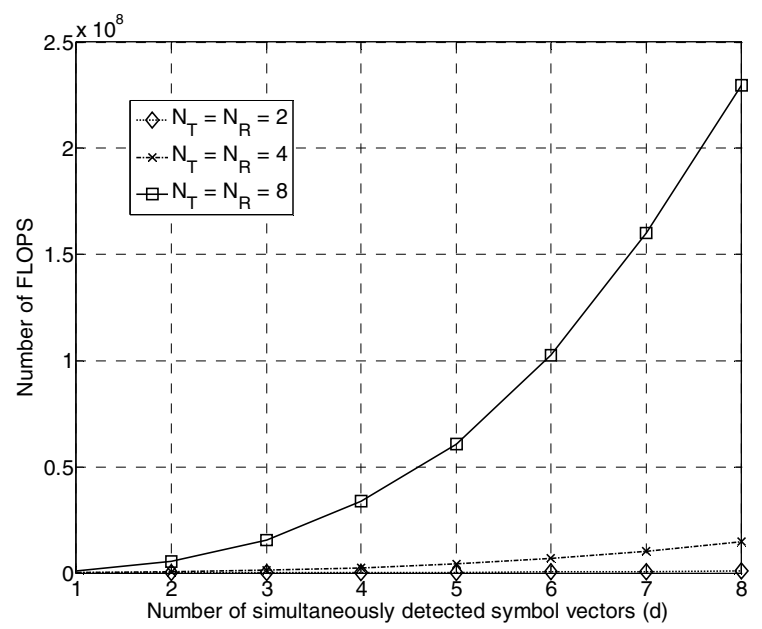

Fig. 4. Comparison of complexity of the PDA-JD and the conventional PDA detectors (the special case of $d=1$ in the PDA-JD detector)

[5] B.M. Hochwald and S. ten Brink, "Achieving near-capacity on a multiple-antenna channel”, IEEE Trans. On Communications, Vol. 51, No.3, pp. 389-399, Mar. 2003.

[6] Baldur Steingrimsson, Zhi-Quan Luo and Kon Max Wong, "Soft Quasi-Maximum-Likelihood Detection for Multiple-Antenna Wireless Channels", IEEE Trans. On Signal Process., Vol. 51, No. 11, pp. 2710-2719, Nov. 2003.

[7] J. Fricke, M. Sandell, J. Mietzner and P. Hoeher, "Impact of the Gaussian Approximation on the Performance of the probabilistic data association MIMO decoder," EURASIP Journal on Wireless Communications and Networking, Vol.5, No.5, pp. 796-800, Oct. 2005.

[8] Feifei Cao, Jiandong Li and Jiawei Yang, "On the Relation between PDA and MMSE-ISDIC," IEEE Signal Process. Lett., Vol. 14, No. 9, pp. 597-600, Sept. 2007

[9] Fredy D. Neeser and James L. Massey, "Proper complex random process with applications to information theory," IEEE Trans. Inf. Theory, Vol. 39, No. 4, pp. 1293-1302, Jul. 1993.

[10] P. W. Wolniansky, G. J. Foschini, G. D. Golden, and R. A. Valenzuela, "V-BLAST: an architecture for realizing very high data rates over the rich-scattering wireless channel," Proc. ISSSE-98, pp. 295-300, Italy, Sept. 1998.

[11] M. K. Varanasi, "Decision feedback multiuser detection: a systematic approach," IEEE Trans. Inf. Theory, Vol. 45, No.1, pp. 219-240, Jan. 1999. 